\title{
California Institute
}

of Integral Studies

Volume 1

Issue 1 Issue 01/2005

\section{Journal of Conscious Evolution}

May 2018

\section{Alchemical Transformation: Consciousness and matter, form and information}

Peat, F. David

Follow this and additional works at: https://digitalcommons.ciis.edu/cejournal

Part of the Anthropology Commons, Cognition and Perception Commons, Cognitive Psychology Commons, Developmental Psychology Commons, and the Philosophy of Mind Commons

\section{Recommended Citation}

Peat, F. David (2018) "Alchemical Transformation: Consciousness and matter, form and information," Journal of Conscious Evolution: Vol. 1 : Iss. 1 , Article 4.

Available at: https://digitalcommons.ciis.edu/cejournal/vol1/iss1/4

This Article is brought to you for free and open access by the Journals and Newsletters at Digital Commons @ CIIS. It has been accepted for inclusion in Journal of Conscious Evolution by an authorized editor of Digital Commons @ CIIS. For more information, please contact digitalcommons@ciis.edu. 


\title{
Alchemical Transformation: Consciousness and matter, form and information
}

\author{
F. David Peat \\ Department of International Relations, Gyengsang National University
}

\begin{abstract}
The paper cautions that seeking rapid resolution of questions involving consciousness and matter may only obscure some of the deeper issues involved. A plea is given for maintaining a certain creative tension or alchemical containment with regard to these issues. Phenomena such as mystical states, projective identification and synchronicities suggest the existence of global mind that transcend quasi-mechanistic theories of consciousness. Alternative approaches based upon the ideas of active information, patterns of form and meaning, and the implicate order are discussed.
\end{abstract}

There has grown up, relatively recently, a nucleus of interests called ìconsciousness studiesî in which physicists and mathematicians, as well as neuroscientists and psychologists, attempt to discover the origins of ìconsciousnessî within the brain. A variety of approaches are employed, some based upon neural networks, others that argue that consciousness must have a quantum mechanical basis, or involve self-organization arising out of non-linearity. A common thread is a certain sense of optimism that ìthe question of consciousnessî will yield its secrets in the same way as, for example, the genetic code or sub-atomic matter.

I must confess that I find none of the approaches or arguments particularly convincing. "It could be soî, I find myself thinking, "but it could equally be otherwise.î My greatest concern is that old habits of thinking and ways of seeing, which could, in part, be termed mechanistic or reductionist abound in this new field, a field, I believe, that is radically different from anything science has hitherto explored. There are deep philosophical problems still to be debated, issues involving questions of identity, the role of the observer, the movement from object to process, the role of the subtle and the transformation of matter by what may lie beyond. We must move cautiously.

The issue is informed from several different sources. There is the subjective, which can be perfectly rational and, in a cer tain sense, scientific. The subjective includes knowledge and approaches from the personal, the psychotherapeutic and various meditative and mystical traditions. Then there is our increasing scientific knowledge of the brain's structure and chemistry, as well as the similarity of certain neurotransmitters to molecules employed by the immune system, which, distributed throughout the body, may well be as complex and equally subtle as the brain itself. Speculations about consciousness, non-locality, subtle levels of matter and active information also come from theoretical physics. Such an influx of creativity, from the theoretical sciences, is to be welcomed yet there is a danger of a certain naivetÈ capable of glossing over much deeper issues.

To this list of sources I would add the philosophical tradition, of West and East, instruments of investigation that are sometimes ignored by scientists working in the field. Anyone speaking about "consciousness", for example, should be cautioned by Wittgenstein's writings on language games. Indeed, language is one of the key issues in our whole venture. Having had the privilege of discussions with Native American elders who speak the Algonquin family of languagesóall strongly verb-basedól realize how deeply conditioned is our thinking by noun-based Indo- European 
languages. Our acts of speech incline us to perceive a world of objects and of concepts. The creation of the concept, placing a boundary around thoughts, ordering them into classes, and classes of classes, seems inevitable to us and the very basis of our logical thought. It is therefore salutary to realize that an equally rational and deeply philosophical people do not involve themselves with the creation of concepts but base their thinking on process, transformation and flux.

Were a Blackfoot to write an essay on the subject of ìconsciousness" (of course this concept itself would never arise) many of the problems that currently face us-would never arise; a different set of difficulties could, to us, be particularly illuminating. in particular, since group consciousness, shared dreams, constant transformation, and participation in a world of ìenergies" or ìspirits,î seems perfectly natural, Blackfoot philosophers may be less interested in ìcollective mindî that in speculating as how such a thing as an individual consciousness and a fixed ego could ever emerge out of such a flux.

In engaging in these investigations we should keep Niels Bohrís maxim before us, iWe are suspended in language in such a way that we cannot say what is up and what is down. We must never forget that ìrealityî too is a human word just like ìwave' or ìconsciousnessî. Our task is to learn to use these words correc tly.î

\section{ALCHEMICAL TENSION}

Our investigation can be approached by a number of different routes or disciplines and we should maintain a creative tension between them, not giving in too quickly to the natural impulse to discover new solutions, propose exhaustive definitions and seek intellectual closure to every problem.

The psychologist Carl Jung gave us the image of the alchemical vessel in which processes of sublimation and purification take place. Psychotherapy provides this same kind of containment whereby a person's tensions and paradoxes are contained within the therapeutic hour, charges with such energy that they may eventually give way to active transformation. Within the alchemical vessel thereís no resolution of paradox and opposition, no compromise, no simple order that ties in between. Rather, a transcendental functions required which moves beyond the limits inherent in different positions by creating new domains. But for this to happen it is necessary to have a period, and a means, of active containment. Even nuclear fusion requires the hot plasma to be contained long enough for fusion reactions to take place.

The same is true of scientific and philosophical ideas. David Bohm regretted the speed with which Niels Bohr attempted to resolve the tensions inherent in quantum theory. Within a year of Heisenbe rgís discovery of matrix mechanics, $\mathrm{Schr}^{\wedge}$ dinger produced his wave equation and Bohr and his colleagues were quick to demonstrate the mathematical equivalence of the two approaches. Yet Heisenberg and $\mathrm{Schr}^{\wedge}$ dinger approached the same problem in subtly different ways-while $\mathrm{Schr}^{\wedge}$ dinger wave equation is written, using Cartesian coordinates, in configuration space, Heisenberg's matrix mechanics makes no reference to an underlying or background space. If only the two approaches had been held in tension, emphasizing both their similarities and differences, Bohr argued, then it may have been possible to develop a much deeper theory, one that transcended conventional notions of space-time and allowed for an intimate connection with relativity. 
A similar tension exists today between scientific approaches to consciousness theory (in which the origin of mind is attributed to objective structures and processes within the brainóalbeit some of them quite novel, such as Penrose's notions of the gravitational collapse of the wave function) and our subjective experience of consciousness, rare moment s of transcendence and those inexplicable occurrences in which the irrational breaks through in dreams, synchronicities, etc. Then there are those phenomena which seem to have a foot in both camps. These include Jungís psychoid which is neither matter nor mind but bot $\mathrm{h}$, the a forementioned synchronicities, and phenomena such as projective identification.

Rather than seeking an immediate resolution between the subjective and objective, it is valuable to hold on to the differences and to the paradoxes, using them as pointers to something deeper. As Wolfgang Pauli put is, now that psychology has discovered the objective within consciousness (Jung's collective unconscious) so too physics. must discover the subjective in matter. He also suggested that physics must come to terms with "the irrational in matterî.

While the problems that face us have been around since the dawn of philosophy, we happen to be in a particularly privileged position today. Science is producing ever more information about subtle structures and electrochemical processes within the brain. Openness to Eastern meditative traditions brings with it alternative theories of consciousness and subtle matter. Transpersonal psychology addresses the idea of collective mind. Quantum theory and chaos theory loosen the appeal of traditional mechanistic theories and reductionistic approaches and, in the process, provide us with new metaphors.

Nevertheless we still remain victim to over two hundred years of mechanistic thinking and we think and communicate with language that reflects and supports such a world view. As soon as we speak about min d or consciousness we find ourselves talking about objects, concepts, things, localization in space, separation, and movement in time. Both quantum theory and Eastern psychology point to timelessness, to active process and the ultimate illusion of the personal observer. Yet, despite the fifty years of its existence, it instill difficult for us, even now, to fully embrace the quantum paradigm, As the physicist Basil Hiley puts it, ìPhysicists come to praise Bohr and decry Einstein (for his adherence to causality and independent elements of reality), yet they end up thinking like Einstein and ignoring Bohr.î

Even the mathematics of quantum theory is still (paradoxically) expressed using space-time coordinates while the theory itself predicts the breakdown of space-time structure. And time itself, as Prigogine points out, has never been treated correctly in physics. Up to now it has been used more as an ordering parameter ëtí, and conveys nothing of the dynamic in which being gives way to becoming.

In this essay, already we find ourselves seduced by details and must constantly remind ourselves that the question is not simply one of dispassionate scientific enquiry. We consider the existence of collective consciousness and what this may mean for world peace. Again we need to maintain that alchemical tension between, on the one hand love and human c ompassion, and, on the other, rational enquiry and clarity of argument and communication. 


\section{LOCALITY AND BEYOND}

Considering the question of collective consciousness I find myself asking: 'What is it that could exist independent of the physical brain?' Yet as soon as I formulate the question I prejudice the answer with concepts of object, location in space and so on. Maybe the most difficult task that faces us is understanding how to formulate the correct question!

Current ìconsciousness studiesî in the hard sciences assumes that mind, or consciousness, evolves and emerges out of the physical brain and cannot therefore exist independent of itóalthough variety of physical signals can be sent between brains. Our experience of consciousness awarenessóscanning the environment and having access to our memoriesóis certainly conditioned by the state of the physical brain. But to suggest that brain is the sole cause of mind does not follow logically.

One school of thought within consciousness studies argues infamous of a quantum mechanical origin for mind. While this may well be the case, I do not find the argument particularly satisfying or logically compelling. In its barest form it proposes that the sort of things done by consciousness (Penrose picks our mathematics) cannot all be reduced to algorithmic processes and therefore min $\mathrm{d}$ cannot have an exclusively mechanical basis. While parts of the brain may be hard-wired it cannot totally operate like a computer or neural net. Quantum theory, the argument goes, is the only other physical domain we know about that cannot be reduced to algorithmic form. Ergo quantum theory must have something to do with consciousness. From this point researchers rush on to theories of quantum tunneling, collapsing wave functions, non-local connections and coherent quantum structures.

But a variety of other approaches are possible:

That mind was present in the universe ab initio. For example, in the form of a proto mind associated even with the elementary particles. The emergence and evolution of complexity occurs in both matter and mind, each perhaps assisting the other.

That mind is of a totally different order from matter, that it cannot be reduced to any scientific, quantitative description animates its liaison with matter via the medium of the brain (The dualism of Popper and Eccles).

That both mind and matter (at the quantum or sub-quantum level) arise out of some deeper level. Or, to follow Bohm, that mind and matter constitute an unanalyzable whole which must therefore be addressed within a totally different order of explanationóthe Implicate Order. In this case the Cartesian cut between matter and $\mathrm{m}$ ind is an illu sion, albeit a very effective and compelling one, that is present only at the Implicate Order of perception, language and explanation.

\section{PHENOMENA}


Before we go too far along this road of invoking various theoretical explanations it is important to return to the direct experience of psyche. The examples below are totally at odds with a scientific and mechanistic description of the world but are held to be true by a variety of traditions, or in psychiatric theory.

\section{a. Projective Identification.}

Projective Identification offers a paradigm case of the tension between, on the one hand, a physics still steeped in mechanistic thinking and the immediacy of psychic experience. Projective identification should be distinguished-from Transference, in which the patient "projects" his or her fantasies (for example, involving authority figures) onto the blank screen of the therapist. While the therapist will be aware of the nature of the projection, these are never internalized (unless counter-transference takes place). In projective identification something more akin to a literal projection of psychic material takes place.

It may happen that, during a session, the therapist experiences, without necessarily being aware that something unusual is going on, memories, feelings attitudes, associations that lie outside his or her experience. At the time, however, these are indistinguishable from ìtrue memories.î It is only later that the therapist realizes that the patient has injected external psychic material into the therapistís mind where, for a time, they had taken root and integrated with the therapist's own personality.

It is very difficult to account for what happens. Clearly some aspect of the patient's psyche-a set of associations or a complex of memories and desires-has fragmented from the self and been projected outwards into the mind of the therapist. In its new location, and for a limited time, it integrates with the therapist's own consciousness to produce awareness of new memories and associations. The patient is now able to view what was previously the very painful contents of personal consciousne ss in an o bjective way for now it belongs to someone else. The final result, hopefully, is to allow this material to be reabsorbed and reintegrated in a more creative manner.

Projective identification appears to be a strategy used by the mind to induce movement and transformation. One thinks of certain chemical reactions which, although energetically advantageous, cannot take place because energy barriers between molecules must first be overcome. Although chemical transformation is desired, it is prevented by these internal energy barriers. Using a catalyst, however, molecules absorb on its surface and then 'borrow" energy needed to undergo the necessary transformations whereby they can react together. After reacting they are then free to leave the catalyst's surface. In Projective Identification the mind of the therapist may play a similar role, allowing certain complexes to be absorbed into anew psychic matrix where they become "free" and more able to undergo creative transformation. Presumably the healthy mind of the therapist possesses something akin to a "psychic immune system" which is able to detect such projected material and eventually reject it so that alien memories do not possess the therapist for too long.

Projective Identification forces us in the position that ìsomethingî is being projected across space, from one mind to the other. This seems a more satisfying explanation than the assumption that both minds have access to some common pool of consciousness-for something seems to be shot, like the darts of a Medicine Person, from one to another. Of course this does not mean than "mind" as such is 
projected. It may simply be some sort of encoded information about mental processes, structure and content that projects from one brain to another. Once in its new location this information activates (like a virus) and makes use of mental energy to form a new cente $r$ within consciousness . (A key question of this essay is how and where such information is encoded.)

I believe that Projective Identification is more common than we assume and may have a much wider purpose than purely neurotic or psychotic relief. It is, for example, the mechanism whereby art operates, is projected outwards and encoded on the surface of a painting as gestures, masses, shapes, colors, and everything else that makes up a "visual code". Likewise music, as the composer Edgar Varese put it, is "the corporealization of thought". The listener or viewer can also "enter into" the work and gain access to this activity of encoded information which then acts to induce transformations of consciousness. The greatest art and music involves a transformation of consciousness and operates at the level of the psychoid. It also deals in what lies beyond the purely personal and enters into the universal and transcendental, the artist no longer projecting individual material but acting as a vehicle.

\section{b. Mystical State}

In such states ego boundaries disappear as the mystic partakes in a transcendental reality, or speaks of having access to ancient knowledge, powerful symbols or alternative realities. Psychiatry may seek to explain this in terms of psychic inflation, access to the archetypes or (following Grof) as an inherited body-memory. Mystics, however, suggest that consciousness opens to the ground of all being. The Indian teacher, J. Krishnamurti proposed that when the brain dies to thought, something else operates that brings about mutation of the brain's structure and a permanent transformation of consciousness. This suggests quite different mechanisms than Projective Identification, for now the mind becomes an aspect of something much larger. This wider entity does not seem to be a Group Mind, as such, but more a non-corporal intelligence or World Soul.

\section{c. Group Mind}

The sharing of consciousness, particularly during dreaming, is a well established phenomenon amongst many Indigenous peoples. Indeed, it is often the basis upon which their society functions and decisions are made. From a pragmatic position within such societies Group Mind must be taken as a fact of life. David Bohm also believed that something similar could be achieved through his 'dialogue process.' (Thirty to forty people meet on a regular basis for many months or even years to dialogue in a free or open way.) Does this imply some new form of communication between individual minds, or does it lie in the ability of each minds to enter into some deeper, collective domain?

\section{d. Paranormal Phenomenon}

There are a variety of anecdotal reports suggesting that individuals can practice remote viewi ng, move objects or have access to other minds. Personally I find it difficult to come to terms with such material. To my mind the experiments are not always convincing, moreover their design and interpretation often seems to me quite mechanistic and the phenomena themselves sometimes appear quite commonplace when compared with the richness of human experience and creativity. 


\section{e. Synchronicities}

During periods of extreme psychic stress experiences, replete with meaning, occur that appear to transcend the boundaries of matter and mind, space, time and causality. The touchstone of such experiences is the deep nature of their meaning. In a sense they are true epiphanies, moments of illumination and possible transcendence, certainly far from mere acts of coincidence. Like (d) above, they remain anecdotal but are, difficult to dismiss. They suggest that mind and matter may be aspects of some underlying realityóJung's psychoid, Bohm's Implicate order.

\section{NON-LOCAL MIND}

How are the various phenomena above to be explained? There are two general lines of approach. The first asserts that something is being transmitted, transferred or projected between two individuals. The second that mind is able to partake, dip into, or unfold out of some common underlying ground that is not bound by the categories of space, time a nd causality.

I do not believe that these need be mutually exclusive. The former may generally operate in, for example, projective identification and hypothetical psychic phenomena while the latter occurs during synchronicities, group minds and manifestations of the psychoid.

\section{a. Transmissions}

Theories of transmission assume that, in addition to speech, pheromones, subliminal gestures, touch, and various hypothetical electromagnetic effects, there exist more subtle forms of transmission.

Yet as soon as one begins with the assumption of interchanges between two spatially located minds then one is firmly based within a mechanical order of space and time. Transmission effects may indeed occur but it is difficult to see how they can account for the richness of all the phenomena discussed above.

\section{b. Fields}

This is currently a popular explanation for the way people appear to share, communicate, or become involved in common thought processes and behaviour. It is an approach that attempts to transcend earlier mechanistic explanations involving senders and receivers of information. Rather, different individuals are supposed to be able to dip into, or even be captured by, this field. Yet one should never forged that a field is, in essence, a notion imported from classical physics. A field carries energy and is defined at each point in space. Hence, as one tries to flee mechanism, one still remains trapped in the language of objects and location.

There are also quantum fields, but these are also defined at all space-time points and can only be stopgap notions on the way to a deeper quantum theory. It may turn out, for example, that there exist non- 
local quantum fields but to my knowledge the mathematics for such a description are yet to be worked out.

Fields may well appear more subtle than Newtonian billiard balls, neverthe less, they stil 1 belong to the world of matter since they are the means by which particles interact with each other. (There are also non4inear fields in which the particles become singularities or solitons of the field itself.) Fields are still very different from minds. Of course, one could attempt to retain the idea of field while progressively dropping the notion of object, space, time, energy, and so on. But in such a case one has really given birth to a radically new concept in science, something which is not, in fact, a field at all.

I am therefore very cautious about using the term 'field" and importing with it a flurry of unexamined assumptions -into the study of consciousness. It may be a short hand way of speaking about whatíre vague and subtle concepts but I cannot see how it helps us understand things in any clearer way since the concept of "field" carries with it much baggage from classical, mechanistic physics. What is called for is a totally new concept. Maybe if we agree to set aside that word "field", on a temporary basis at least, we will be forced to face phenomena themselves and seek some more appropriate concept.

\section{c. Non-local connections.}

Quantum theory (Bell's Theorem) permits non-local connections and at first sight this is sometimes taken as the mechanism for communication between minds, or evidence of "non-local mind". But, it must be emphasized, these quantum correlations cannot be used to carry signals or information. Neither can quantum connections be invoked to explain paranormal phenomena.

This is not to deny that non-local connections may indeed exist between brains. Or that mind transcends the distinctions of space and time. But this cannot be justified by appealing to Bellís Theorem. It may, however, be useful to propose that Bell's non-local connections are themselves a special case of something more general. Non-locality may, for example, be the direct consequence of global forms, forms that are not defined within conventional space-time. In view of the importance of form in biological systems, in the Jungian archetypes, and as the "form of the wave function", the role of form in consciousness may be a p rofitable route to explore.

\section{d. Information}

An idea that escapes from the associations connected with "fields" into the domain of the subtle is that of information. It is a notion that is beginning to surface within the physical sciences, immunology, and even psychotherapy. Indeed, the argument could be made that just as during the nineteenth century science moved from an exclusive focus upon matter, its movement and transformations into a consideration of energy in its various aspects, so too today physics will incorporate on an even more subtle level the notion of information.

Hitherto information has been treated in a somewhat subjective way as something exterior or peripheral to physical law. An increase in entropy could be equated with a decrease in order, or information about a system. Yet there were always difficulties connected with the definition of entropy. Shannon and Weaver's "Information Theory" was more concerned with redundancy and 
degradation of information than with its particular content. Perhaps the first objective use of information came with Beckenstein's discovery that the radius of a Black Hole is related to its entropy or information content.

Yet over the past years there has been an increasing appeal to the notion of information. Rupert Sheldrake's "morphoge netic fields" are supposed to "guide" the development of everything from crystals and organism to instincts and learning. Since these hypothetical fields contain or store the memories, or habits, of matter and consciousness they could indeed be called repositories of information. The drawback of Sheldrake's approach is that the nature of existence of these fields or their means of operation on matter and consciousness is not spelled out.

Ervin Laszlo had also proposed a reservoir of information in what he terms the psi field. This information appears to contain, amongst other things, the totality of what a person has thought, said and done. In this sense human minds would, in principle, have access tithe totality of human knowledge. The existence of the field itself appears to be equated with a proposed scalar spectrum of the vacuum field of quantum theory. The present author has not studied Laszlo's work to such an extent that he could make any definitive comments on the theory.

David Bohm has also introduced the notion of information in his Ontological Interpretation of quantum theory and extended this to speculations on the immune system and the nature of consciousness. His notion of "active informationî will be discussed below.

There are obvious advantages of beginning with the concept of information since, because it partakes of $b$ oth, it offers the possibility of tr anscending the traditional division between subjective and objective, matter and mind. The major difficulty lies in its ontology. What exactly is the nature of its existence and in what form is it present in mind and matter? If, for example, one talks of 'fields of information" then one is back again to the old Cartesian world of objects in space and the need for a medium for the transmission of signals. If information is to prove useful then it has to have its own new level of description and its own mode of existence. Clearly, information demands mathematical forms that lie beyond the concept of field. Maybe, for example, information is born at the level of prepack.

There is also the question of what is being encoded and how is this done. A clue to the nature of encoding can, I believe, come from art. Over centuries and even millennia, artists have developed visual codes in which to convey such things as mimesis, numinous power, emotional force, movement and the like. Similar codes exist in music. Such codes lie far from a 1:1 mapping. They are more subtle since acts of encoding and decoding rely upon a deep intuitive understanding of the human perceptual system as well as upon the history of art, human symbolism, and the nature of particular societies.

To take but one example about perception. Matiss e began with fairly detailed drawings of the female figure which were progressively ìsimplified,î in the sense of a decreasing density of marks upon the page. The final drawing may have the appearance of extreme simplicity yet the placing of each line, its relationships to others, the nature of its gesture, and so on, all convey a surprising amount of information. In a certain sense the most successful could be said to have a higher iinformation 
content.î Yet where does this information lie? Certainly not on the page alone, in the sense of number of bytes of information. Rather in involves the activity of perception on the part of the human viewer, in his or her engagement with the work and knowledge of visual codes. Art, in this sense, becomes a dialogue over the centuries through the medium of visual codes. It is a discussion of painting through painting.

It is my conjecture that, within the context of consciousness studies, 'information' is also of this type. It is not a simple representation of quantitative data-mass, position, momentum and so on, nor it is a mere digital read out or what was said and done. Rather it must be some sort of 'artistic' encoding that requires the active participation of human consciousness to reactivate. In this sense a mathematics of the code would have to be developed that specifically incorporated the activity of consciousness in its interaction and activation of information. One could also speculate that this process of activation is not confined to human consciousness but may pervade the cosmos.

\section{e. Active Information and Prespace.}

Bohm's Ontological Interpretation of quantum theory p roposes that, at one 1 evel, the electron is a particle guided by a quantum potential. (At a deeper level Bohm speculated that the electron is a process that takes place in some hypothetical prespace.) In seeking to explain the quantum measurement problem Bohm also introduced what he called ìactive informationî to explain the relationship between the electron and the quantum potential.(Essentially the quantum potential and the electron are aspects of an indivisible whole but, for the purposes of presenting his theory, Bohm treated them as separate.)

Bohm proposed that quantum processes are guided by information-not passive encoded data but an actual activity of information. Bohm's analogy is with the way subtle information in a television signal impresses itself upon, and thus gives form to, the crude energy entering the electrical plug. In this way a subtle signal can give rise to pictures and sounds. For Bohm, the activity of information acts on both matter and energy. He also speculated about active information within the immune system-a form of intelligence delocalized over the whole b ody. Thus, for Bohm, a change of meaning in the mind becomes a change of actual being in the body.

Bohm's hypothesis is important, I believe, for the following reason. It is not so much that the Ontological Interpretation may or may not be correct but rather that it is a formal, mathematical system (one that can be used to make calculations and predict result) that incorporates the notion of information. Hence information has been brought into physics in an entirely objective fashion. Yet, having said this, it is clear that a number of serious problems remain. What exactly is the ontological status of active information? How is it to be described? Where does it exist? The whole topic is exciting but requires much work. It is tied to other speculative ideas in quantum theory about structures that exist prior to space and matter.*

Prespace algebras, such as the Grassman algebra, begin with a fundamental distinction in a featureless ground. Thought is indivisible yet creative perception can distinguish opposite poles in thought. Once the first perception, or distinction, has been established then the algebra begins to generate itself and in the process develop structures that may be the precursor of space. In an analogous fashion a 
thought in the mind is created out of an instant of perception. Onc e this perception has taken place and the thou ght is born, then it begins to move by a dialectical process-in this way psychological time is born. Both space and thought seem to be born out of an analogous timeless, creative process.

\section{f. Form}

Another approach to thinking about information is through the notion of "form". Form is a key concept in biology. The function of everything from the activity of an enzyme to a cell or organ is relate to its physical form. Growth from the fertilized cell to the adult is a process of differentiation and transformation of form; hence biologists from Aristotle to Waddington, Sheldrake and Goodwin have postulated notions of ìmorphic fields.î

\footnotetext{
* As to the encoding of information at the quantum level, important clues may come from the reduced density matrix (a 2-particle form which contains information about the total N-particle wave function). N-particle information is encoded, or enfolded, within the reduced density matrix (as it is in Green's Function). The $\mathrm{N}$-representability problem deals with the question of how the antisymmetric form of the wave function places restrictions on the form of the reduced density matrix. It should be recalled that quantum non-locality exists precisely because of the antisymmetric form of the wav e function-an antisymmetric form cannot be facto rized into subcomponents. Hence there is a deep connection between non-locality, information and the form of the density matrix. A further connection is that $\mathrm{N}$-representability conditions are connected to Grassman algebras (the algebras of exterior forms) and it is precisely than same algebras that seem to hold the key to prespace. Such a rich interconnection of ideas cannot be mere coincidence.
}

Form has associated with it the idea of a Gestalt, of global patterns, perception and non-locality; such notions may well connect with the functioning of consciousness and with the immune system. The universal importance of form and its transformation was, in the 1960s, the subject of a new branch of mathematics, RenÈ Thom's Catastrophe Theory.

A particularly important aspect of form is its global nature, since it deals with changing shapes, shapes that must be seen in a gestalt manner, shapes that are entire and complete in themselves. Dealing with form is the reverse of trying to build up larger structures from individual elements, or describing space in terms of coordinates. Form seems to be an ideal way of dealing with the collective and distributed.

Form also has a role to play in physics. In classical physics it is the form of the Hamiltonian that remains invariant under canonical transformations. In this way, Newtonian mechanics can be transformed from the mechanical interaction of individual particles into global form-preserving processes. Likewise, General Relativity is about the invariance of form under all possible coordinate transformations. In this sense, motion under gravity has to do with the preservation of a certain form. One could perhaps generalize the concept of inertia to that of the ìlaw of persistence of form.î

Most dramatically, form appears in the guise of the wave function. It is the global form of the wave function (symmetric or antisymmetric) that is responsible for the existence of Fermi-Dirac or BoseEinstein statistics. The fact that such forms are non-factorizable (into spatially independent components) is the deep reason for quantum non-locality (Bellís mysterious correlation between distant particles). The global form of the wave function is ultimately responsible for collective modes in physics-plasma, superfluid, superconductor and hypothetical Frochlich systems. The form of the wave function orchestrates each of an astronomical number of particles into a highly coordinated dance. 
Bohm's quantum potential also connects with the notion of form. As a potential it is unique in physics since the nature of its effect on the motion of an electron does not arise f rom its strength or intensity but from its formóits part icular complex shape. The quantum potential is inherently nonlocal, its effects do no fall off with distance and for this reason quantum objects, well separated in space, can remain strongly correlated.

Form could also have something to do with global (quantum) processes within the brain that give rise to consciousness. As a global property it may be relevant to the evolution of space-time structure out of some more primitive quantum prespace. Penrose, for example, proposes that the quantum mechanical ìcollapse of the wave functionî is a global phenomenon connected with the geometrical properties of space-time. He also speculates that global quantum processes have a role to play in the liaison between consciousness and brain structure.

\section{g. Meaning}

If form begins with biology (and leads into quantum theory), meaning surely starts in psychology. It was Carl Jung who stressed the role of meaning in Synchronicityóthat region where form and pattern spill over the boundaries between mind and matter. For Jung the key was the deep internal significance associated with an experience of synchronistic patterns, a significance that does not end at the boundaries of personal consciousness. Meaning therefore is both subjective and objective. Jung termed this speculum between matter and mi nd the ìp sychoidî; its integrating fac tor is meaning.

In t he context of Dialogue, Bohm spoke of a "field of meaning" shared by all participants. He stressed that the way to bring about effective social change is through an overall change of meaning. Meaning, which could be thought of as a field of form; Bohm associated this with the immune system. The immune system is what keeps the body whole, coordinates the processes of the body and maintains its level of meaning. If this meaning is somehow degraded then the body becomes sick.

Bohm stressed that his maxim 'a change of meaning is a change of being" was to be taken literally. An assailant seen on a dark night turns out to be the shadow of a tree trunk. Immediately a flurry of electrochemical changes takes place in mind and body. Laboratory research suggests that shifts in 'meaning" bring about subtle restructuring of nerve pathways and the sensitivities of connections. Meaning, which is normally taken to be subjective, turns out to have an objective, physical consequence. Meaning can act on matter and, presumably, matter on meaning.

\section{IMPLICATE ORDERS}

Discussions of mind and matter constantly run up against limitations of language and formalism. Much of our thinking and language is based in a world of space, time and causality. Bohm termed this the Explicat e Order and proposed that a radically different Implicate, or Enfolded Order exists. Within this order the duality of matter and mind obtain their resolution. What appear as distinct objects, well separated in space and time are, within the Implicate order, enfolded each within the other. What at one level appears as object at another becomes process. 
While personal consciousness appears to be attached to an individual brain and body, Carl Jung proposed the collective unconscious which contains material shared by all people in the form of powerful symbols and archetypical forms. Even deeper than the collective lies the psychoid which partakes of both matter and mind while transcending their distinction. It is at the level of the psychoid that synchronicities occur and at the level of the collective that an archetype may become activated across a whole society.

Jung's ideas are seductive, yet again the nature and ontological status of the collective is far from clear. Maybe this is why the shift offered by the Implicate Order is so attractive. Ontology attempts to answer questions about an object's status of existence. But it may note useful, at this junction, to keep enquiring about the nature of the existence of mind, or information, or the psychoid. It is here that Bohm's Implicate Order offers a new way of proceeding for it offers desc ription, and a perception, which escapes mechanistic thinkin g. Questions of transmission, localization and delocalization simply do not arise within the Implicate Order since it ties beyond the categories of space and time.

Instead of talking about object we deal in process, we enquire as to how a particular explicate (individual mind/body) unfolds out of the Implicate. Minds become both truly collective and personal by virtue of the continuous process of unfoldment and enfoldment whereby they are united within the Implicate and individuated within the Explicate. Mind and matter are connected because of their essential identity within the Implicate Order.

\section{Acknowledgements}

This enquiry has encouraged and illuminated on two fronts, discussions with Basil Hiley at the Physics Department, Breakneck College and with Christopher Hauke, a Jungian therapist.

KEYWORDS: consciousness, non-locality, global mind, projective identification, synchronicity, prespace, implicate order

World Future, 1997, Vol. 48. pp. 3-22. (C) 1997 OPA (Overseas Publishers Association).

Reprinted with permission. Reprints available directly from the publisher, Amsterdam B. V..

Published in The Netherlands under license by Gordon and Breach Science Publishers.

Photocopying permitted by license only. Printed in Malaysia. 